\title{
AVALIAÇÃO DO GRAU DE INTELIGIBILIDADE DE FALA DE CRIANÇAS COM DESVIO FONOLÓGICO: IMPLICAÇÕES NAS HABILIDADES SOCIAIS
}

\author{
Assessing speech intelligibility level of children \\ with phonological disorders: implications in social skills
}

Fabiana Cristina Carlino ${ }^{(1)}$, Almir Del Prette (2), Dagma Venturini Marques Abramides ${ }^{(3)}$

\begin{abstract}
RESUMO
Objetivo: analisar a relação entre o grau de inteligibilidade de fala e as habilidades sociais de comunicação em crianças com diagnóstico de desvio fonológico. Método: participaram deste estudo 10 crianças, diagnosticadas com desvio fonológico, com idade cronológica média de 7,28 anos, sendo três do gênero feminino e sete do masculino, que realizavam terapia de linguagem duas vezes por semana em uma clínica escola de uma cidade de médio porte do estado de São Paulo. As crianças foram submetidas à avaliação da fonologia por meio do instrumento ABFW, sendo que a gravidade do desvio fonológico foi baseada no cálculo do Percentual de Consoantes Corretas (PCC). E em seguida foram filmadas em situações estruturas de interação de maneira tal a obter uma amostra de fala espontânea, para caracterizar o grau de inteligibilidade de fala (GIF), além de observar presença ou ausência dos componentes das habilidades sociais de comunicação (HSC). Para analisar a relação entre GIF e HSC foi utilizado o coeficiente de Spearman. Resultados: foi possível caracterizar as classes de HSC que encontram-se deficitárias em crianças diagnosticas com desvio fonológico, além de verificar uma relação significante e positiva entre o GIF e o HSC, mostrando que quanto maior o GIF maior a dificuldades nas HSC. Conclusão: o estudo verificou a relação entre GIF e HSC, além de identificar as classes de HSC que encontram-se deficitárias em crianças com diagnóstico de desvio fonológico, favorecendo a elaboração de instrumentos para avaliação e intervenção, permitindo assim, um melhor planejamento para cada caso.
\end{abstract}

DESCRITORES: Comunicação; Desenvolvimento da Linguagem; Linguagem Infantil; Relações Interpessoais

\section{INTRODUÇÃO}

A linguagem representa um papel fundamental na interação do ser humano com o meio, permitindo

(1) Fonoaudióloga da Universidade Federal de São Carlos UFSCar; Mestre e Doutoranda em Educação Especial pela Universidade Federal de São Carlos - UFSCar.

(2) Psicólogo; Professor Titular (voluntário) vinculado ao programa de Pós-Graduação em Educação Especial e em Psicologia da Universidade Federal de São Carlos - UFSCar, São Carlos, SP.

(3) Psicóloga; Professora Livre-docente do Departamento de Fonoaudiologia da Faculdade de Odontologia de Bauru, Universidade de São Paulo - FOB - USP, Departamento de Fonoaudiologia, Bauru - SP.

Conflito de interesses: inexistente ao indivíduo estruturar o seu pensamento, traduzir o que sente, expressar o que já conhece e se comunicar com os demais. A linguagem é considerada a primeira forma de socialização da criança, e, na maioria das vezes, é efetuada explicitamente pelos pais por meio de instruções verbais durante atividades diárias. Dessa forma, a criança tem acesso, antes mesmo de aprender a falar, a valores crenças e regras, entrando em contato com os conceitos de sua cultura ${ }^{1}$.

A aquisição e desenvolvimento da linguagem ocorrem normalmente quando não há alterações nos requisitos biológicos, afetivos e ambientais. Diferentes níveis lingüísticos participam desse processo, dentre os quais destaca-se o nível fonológico ${ }^{2-5}$. Qualquer alteração em um dos requisitos 
pode resultar em uma inadequação no processo de aprendizagem da linguagem, podendo levar, entre outras alterações, ao chamado desvio fonológico6-10.

O sistema fonológico de uma língua constitui o conjunto de seus fonemas, isto é, um grupo relativamente pequeno de sons empregados com valor distintivo. Quando se trata da fonologia, são estudados os fonemas, a maneira como eles se organizam e relacionam-se, além das regras a que estão sujeitos, a fim de formarem unidades lingüísticas maiores, como as sílabas e as palavras ${ }^{11,12}$. O desvio fonológico é uma alteração de linguagem caracterizada pelo uso inadequado dos sons conforme a idade e variações regionais, que podem envolver erros na produção, percepção ou organização dos sons, podendo ser observado em casos de crianças que não aprendem um ou vários sons esperados para sua idade ${ }^{13-15}$. Essa alteração pode ocorrer com o grau de leve a severo em, aproximadamente, dois a três por cento das crianças entre quatro a sete anos de idade, sendo sua ocorrência mais freqüente nas formas mais leves ${ }^{16}$.

No caso de crianças com desvio fonológico, os vínculos sociais podem ser dificultados devido à inteligibilidade de fala ${ }^{12}$. Esse tipo de experiência pode influenciar eventos interpessoais em geral, implicando os modos de constituição de futuras relações sociais ${ }^{17}$.

Algumas pesquisas ${ }^{8,10,18,19}$ mostraram ser possível quantificar a gravidade do desvio fonológico, sendo considerado como mais um recurso avaliativo a ser utilizado no tratamento com base fonológica.

A inteligibilidade pode ser prejudicada ou completamente ininteligível na presença de processos fonológicos (PF: constituem mudanças sistemáticas de som que afetam uma classe de sons ou uma seqüência de sons. Os processos são descrições de padrões que ocorrem regularmente, observados na fala da criança e que operam com o objetivo de simplificar os sons alvos do adulto ${ }^{20}$ na fala da criança. A fala de crianças com desvio fonológico pode ocasionar um inventário fonético restrito, além de um sistema fonológico simplificado que poderá levar a uma fala ininteligível ocasionando problemas no ato de comunicar-se. Portanto, a interação social poderá ser prejudicada, visto que os desvios ocasionarão diminuição da inteligibilidade ${ }^{12,18,21,22}$. Nesse contexto, o objetivo do presente estudo foi analisar a relação entre o grau de inteligibilidade de fala e as habilidades sociais de comunicação em crianças com diagnóstico de desvio fonológico.

\section{MÉTODO}

Nesta pesquisa, foram incluídas crianças que apresentavam diagnóstico de desvio fonológico, na faixa etária entre seis e oito anos e onze meses, que estivessem matriculadas na primeira, segunda ou terceira séries do ensino fundamental regular, público ou privado, que não apresentasse deficiência auditiva, deficiência mental, síndromes, distúrbios abrangentes do desenvolvimento e lesões neurológicas adquiridas.

Participaram deste estudo 10 crianças com idade cronológica média de 7,28 anos, sendo três do gênero feminino e sete do masculino que realizavam terapia de linguagem duas vezes por semana em uma clínica escola de uma cidade de médio porte do estado de São Paulo.

As crianças foram submetidas à avaliação da fonologia por meio do instrumento $\mathrm{ABFW}^{23}$, sendo que a gravidade do desvio fonológico foi baseada no cálculo do Percentual de Consoantes Corretas (PCC), o qual foi obtido pela divisão do Número de Consoantes Corretas (NCC) pelo Número de Consoantes Corretas (NCC) adicionado ao Número de Consoantes Incorretas $(\mathrm{NCl})$, multiplicado por cem. A partir do resultado do PCC, o desvio fonológico das crianças participantes pôde ser classificado como severo (PCC < 50\%), moderadamentesevero $(50 \%<$ PCC < $65 \%)$, levemente-moderado $(65 \%<$ PCC < $85 \%)$ e leve $(85 \%<\text { PCC < } 100 \%)^{18}$. No índice PCC para cada sujeito foi considerado como erro as omissões, as substituições e as distorções comuns e incomuns.

Em seguida, as crianças foram filmadas em situação estruturada de interação com um adulto desconhecido do gênero feminino, de maneira tal a obter uma amostra de fala espontânea, além de observar presença ou ausência dos componentes verbais de conteúdo (fazer e responder perguntas; iniciar, manter e encerrar conversação), verbais de forma (duração, volume, entonação) e não verbais (contato visual, gestos, sorriso, expressões faciais, movimentos de cabeça, postura corporal) das Habilidades Sociais de Comunicação. Dois juízes (fonoaudiólogos) treinados analisaram as filmagens das crianças frente à situação de interação e registraram o Grau de Inteligibilidade de Fala (GIF) e Habilidades Sociais de Comunicação (HSC) no Protocolo de Registro da Avaliação do Desempenho - Filmagem (PRAD - F) elaborado pela pesquisadora.

Para a mensuração das categorias de Habilidades Sociais de Comunicação, foi utilizada uma escala com três pontuações: 
1 - Insatisfatório (I): Quando o participante apresenta 1 dos 4 critérios;

2 - Nem Satisfatório Nem Insatisfatório (NSNI): Quando o participante apresenta 2 dos 4 critérios;
3 - Satisfatório (S): Quando o participante apresenta pelo menos 3 dos 4 critérios de definições da categoria.

\begin{tabular}{|c|c|c|}
\hline Categoria & Definições & Juiz (X) / Juiz (2) \\
\hline $\begin{array}{c}\text { Iniciar } \\
\text { conversação }\end{array}$ & $\begin{array}{l}(\mathbb{X}) \text { Dirige-se ao interlocutor a guisa de "puxar } \\
\text { conversa"; } \\
(\mathbb{X}) \text { Apresenta expressões faciais que demonstram } \\
\text { interesse com variações na fisionomia (olhos, boca, } \\
\text { sobrancelha); } \\
\text { (3) Mantém contato visual com breves interrupções; } \\
\text { (4) Utiliza tom de voz audível para o interlocutor. }\end{array}$ & $\begin{array}{c}\text { Apresenta } 2 \text { critérios da } \\
\text { definição da categoria, } \\
\text { portanto, essa categoria } \\
\text { classifico como NSNI }\end{array}$ \\
\hline
\end{tabular}

Figura 1 - Exemplo das Categorias de Habilidades Sociais de Comunicação presente no protocolo de observação

As possíveis marcações quanto à inteligibilidade da fala eram:

1 - Insuficiente (incompreensível), quando a maior parte das palavras não foi compreensível e teve dificuldade em compreender o tópico principal da mensagem;

2 - Regular (pouco compreensível), quando foi possível compreender pelo menos metade das palavras e conseguiu compreender o tópico principal da mensagem e

3 - Boa (compreensível), quando foi possível compreender praticamente todas as palavras e entender o conteúdo da mensagem.

A fidedignidade interobservadores foi calculada segundo a fórmula abaixo ${ }^{24}$

$$
I C=\frac{\Sigma A}{\Sigma A} \overline{\Sigma D} \times 100
$$

Legenda: IC (Índice de Concordância), $\Sigma$ A (somatória dos acordos), $\Sigma \mathrm{D}$ (somatória dos desacordos)

Foram considerados fidedignos os dados com, no mínimo, $75 \%$ de concordância.

O projeto desse estudo foi submetido ao Comitê de Ética em Pesquisa com Seres Humanos da Faculdade de Odontologia de Bauru da Universidade de São Paulo, recebendo aprovação de número 54/2008 e todos os responsáveis pelos participantes assinaram um termo de consentimento livre e esclarecido concordando com as normas do estudo.

Para verificar a associação entre as variáveis, Grau de Inteligibilidade de Fala (GIF) e Habilidades Sociais de Comunicação (HSC) foi utilizada a correlação de Spearman. O coeficiente de correlação $r_{s}$ linear é um número puro que varia de -1 a +1 e sua interpretação dependerá do valor numérico e do sinal, como segue:

$r_{s}=-1$ correlação perfeita negativa

$-1<r_{\text {s }}<0$ correlação negativa

$r_{s}=0$ correlação nula

$0<r_{s}<1$ correlação positiva

$r_{s}=1$ correlação perfeita positiva

$0,2<r_{s}<0,4$ correlação fraca*

$0,4<r_{s}<0,7$ correlação moderada*

$0,7<r_{s}<0,9$ correlação forte*

*possui o mesmo significado para os casos negativos ou positivos.

\section{RESULTADOS}

$\mathrm{Na}$ Tabela 1 são apresentados os resultados referentes ao Grau de Desvio Fonológico de acordo com a idade e o gênero dos participantes.

Com relação ao gênero feminino foi possível observar a variação de leve a levemente-moderado, enquanto que no gênero masculino a variação foi de leve a severo. Com relação à idade, observou-se que quanto maior a idade menor o grau de Desvio Fonológico.

$\mathrm{Na}$ Tabela 2 são apresentados os resultados descritivos quanto a relação encontrada entre o GIF e o desempenho em HSC observada pelos juízes. De acordo com o coeficiente da correlação de Spearman $\left(r_{s(0,05 ; 10)}=0,648 ; p=0,90\right)$, a correlação entre o GIF e HSC foi considerada moderada e positiva. 
Tabela 1 - Caracterização das crianças quanto ao Gênero, Idade e Grau de Desvio Fonológico

\begin{tabular}{|c|c|c|c|c|c|c|c|}
\hline & \multicolumn{4}{|c|}{ Gênero } & \multirow{3}{*}{$\begin{array}{l}\text { Idade } \\
\text { Média }\end{array}$} & \multirow{2}{*}{\multicolumn{2}{|c|}{$\begin{array}{l}\text { Gravidade } \\
\text { do Desvio }\end{array}$}} \\
\hline & \multicolumn{2}{|c|}{$\mathbf{F}$} & \multicolumn{2}{|c|}{ M } & & & \\
\hline & $\mathbf{N}$ & $\%$ & $\mathbf{N}$ & $\%$ & & $\mathbf{N}$ & $\%$ \\
\hline Leve & 1 & 10 & 1 & 10 & 8,0 & 2 & 20 \\
\hline $\begin{array}{l}\text { Levemente- } \\
\text { Moderado }\end{array}$ & 2 & 20 & 2 & 20 & 7,25 & 4 & 40 \\
\hline $\begin{array}{c}\text { Moderadamente- } \\
\text { Severo }\end{array}$ & 0 & 0 & 2 & 20 & 7,5 & 2 & 20 \\
\hline Severo & 0 & 0 & 2 & 20 & 6,5 & 2 & 20 \\
\hline Total & 3 & 30 & 7 & 70 & - & 10 & 100 \\
\hline
\end{tabular}

Tabela 2 - Grau de Inteligibilidade de Fala relacionada às Habilidades Sociais de Comunicação

\begin{tabular}{c|cccccc}
\hline \multirow{2}{*}{ GIF HSC } & \multicolumn{2}{c}{ Insatisfatório } & \multicolumn{2}{c}{$\begin{array}{c}\text { Nem Insatisfatório } \\
\text { Nem Satisfatório }\end{array}$} & \multicolumn{2}{c}{ Satisfatório } \\
\cline { 2 - 7 } & $\mathbf{N}$ & $\%$ & $\mathbf{N}$ & $\%$ & $\mathbf{N}$ & $\%$ \\
\hline Insuficiente & 1 & 10 & 1 & 10 & 0 & 0 \\
Regular & 3 & 30 & 2 & 20 & 1 & 10 \\
Boa & 0 & 0 & 1 & 10 & 1 & 10 \\
\hline
\end{tabular}

Ainda por meio da Tabela 2, pode-se observar que a maioria dos participantes avaliados com Grau de Inteligibilidade de Fala Insuficiente (GIF-I) ou Regular (GIF-R) apresentou desempenho em Habilidades Sociais de Comunicação Insatisfatório (HSC-I) ou Habilidades Sociais de Comunicação Nem Satisfatório Nem Insatisfatório (HSC-NINS). Apenas um participante classificado GIF-R, apresentou desempenho em Habilidades Sociais de Comunicação Satisfatório (HSC-S).

A Tabela 3 classifica os participantes quanto ao Grau de Desvio Fonológico, Grau de Inteligibilidade de Fala, Desempenho em Habilidades Sociais de Comunicação (HSC) e Dificuldades nas classes de HSC.

\section{DISCUSSÃO}

A classificação do Desvio Fonológico mais utilizada em pesquisas nacionais e internacionais é de caráter quantitativa ${ }^{15,18,25,26}$. Neste trabalho, a análise utilizada foi o Percentual de Consoantes Corretas (PCC) $)^{18}$. Observou-se que as crianças do gênero masculino apresentaram um maior grau de Desvio Fonológico. Esse resultado está de acordo com grande parte dos estudos da área 1, 15, 26-31, uma vez que essa alteração é mais freqüente em crianças desse gênero. Acredita-se que esse fato pode estar relacionado a fatores neurológicos (a maturação cerebral é mais lenta nos meninos), hormonais (os níveis alterados de testosterona poderiam dificultar a realização de conexões ideais pelo sistema nervoso), genéticos e sociais (as cobranças do meio social são mais frequentes e intensas com os meninos, exigindo-se que sua fala seja sempre correta) ${ }^{32}$.

Notou-se também que houve uma relação inversamente proporcional da idade cronológica da criança com o Desvio Fonológico. Isso confirma os dados de que à medida que a criança adquire maturidade funcional, as omissões, substituições e distorções são superadas, fazendo com que sua fala se aproxime à do adulto ${ }^{19,33,34}$.

Por meio da Tabela 2 é possível observar que os participantes que foram avaliadas com Grau de Inteligibilidade de Fala Insuficiente ou Regular foram os que mais apresentaram dificuldades quanto ao Desempenho Social durante a situação estruturada de interação. A fala de crianças com Desvio Fonológico pode ocasionar em um inventário fonético restrito além de um sistema fonológico simplificado que poderá levar a uma fala ininteligível ocasionando problemas no ato de comunicar-se. Dessa maneira, a interação social poderá ser prejudicada, já que os desvios ocasionarão diminuição da inteligibilidade ${ }^{12}$. 
Tabela 3 - Classificação dos Participantes quanto ao Grau de Desvio Fonológico, Grau de Inteligibilidade de Fala, Desempenho em Habilidades Sociais de Comunicação e Dificuldades nas classes de HSC

\begin{tabular}{|c|c|c|c|c|}
\hline \multirow[b]{2}{*}{ Participantes } & \multicolumn{4}{|c|}{ Classificação } \\
\hline & $\begin{array}{c}\text { Grau Desvio } \\
\text { Fonológico }\end{array}$ & $\begin{array}{c}\text { Grau de } \\
\text { Inteligibilidade } \\
\text { de Fala } \\
\end{array}$ & $\begin{array}{l}\text { Habilidades } \\
\text { Sociais de } \\
\text { Comunicação } \\
\end{array}$ & $\begin{array}{l}\text { Dificuldades nas } \\
\text { classes de HSC }\end{array}$ \\
\hline P1 & Leve & Boa & Satisfatório & - \\
\hline P2 & $\begin{array}{l}\text { Moderadamen } \\
\text { te severo }\end{array}$ & Regular & $\begin{array}{c}\text { Nem Satisfatório } \\
\text { Nem } \\
\text { Insatisfatório }\end{array}$ & $\begin{array}{c}\text { Iniciar, manter e } \\
\text { finalizar conversação }\end{array}$ \\
\hline P3 & Leve & Boa & Satisfatório & - \\
\hline P4 & $\begin{array}{l}\text { Levemente } \\
\text { Moderado }\end{array}$ & Regular & $\begin{array}{c}\text { Nem Satisfatório } \\
\text { Nem } \\
\text { Insatisfatório }\end{array}$ & $\begin{array}{l}\text { Fazer e responder } \\
\text { perguntas; }\end{array}$ \\
\hline P5 & $\begin{array}{l}\text { Moderadamen } \\
\text { te Severo }\end{array}$ & Regular & Insatisfatório & $\begin{array}{c}\text { Iniciar, manter e } \\
\text { finalizar conversação; } \\
\text { Responder perguntas; } \\
\text { Contato visual. } \\
\end{array}$ \\
\hline P6 & Severo & Insuficiente & Insatisfatório & $\begin{array}{c}\text { Iniciar, manter e } \\
\text { finalizar conversação; } \\
\text { Fazer e responder } \\
\text { perguntas; } \\
\text { Contato visual. } \\
\end{array}$ \\
\hline P7 & $\begin{array}{l}\text { Levemente } \\
\text { Moderado }\end{array}$ & Regular & $\begin{array}{c}\text { Nem Satisfatório } \\
\text { Nem } \\
\text { Insatisfatório } \\
\end{array}$ & $\begin{array}{c}\text { Iniciar, manter e } \\
\text { finalizar conversação. }\end{array}$ \\
\hline P8 & $\begin{array}{r}\text { Levemente } \\
\text { Moderado }\end{array}$ & Regular & Satisfatório & - \\
\hline P9 & Severo & Insuficiente & Insatisfatório & $\begin{array}{c}\text { Iniciar, manter e } \\
\text { finalizar conversação; } \\
\text { Fazer pedidos; } \\
\text { Contato visual; } \\
\text { Expressões faciais. }\end{array}$ \\
\hline P10 & $\begin{array}{l}\text { Levemente } \\
\text { Moderado }\end{array}$ & Regular & $\begin{array}{c}\text { Nem Satisfatório } \\
\text { Nem } \\
\text { Insatisfatório }\end{array}$ & $\begin{array}{c}\text { Iniciar manter e finalizar } \\
\text { conversação; } \\
\text { Fazer perguntas; } \\
\text { Pedir informação. }\end{array}$ \\
\hline
\end{tabular}

Foi observado por meio do coeficiente da correlação de Spearman $\left(r_{S(0,05 ; 10)}=0,648 ; p=0,90\right)$, uma correlação entre o GIF e as HSC, estatisticamente moderada e positiva. Demonstrando que quanto menor o GIF menor o desempenho em HSC, o que permite identificar que as crianças que tiveram maior dificuldade com relação à emissão verbal apresentaram igual dificuldade em interagir de maneira socialmente habilidosa. As crianças com Desvio Fonológico apresentam dificuldades relacionadas aos vínculos sociais devido à inteligibilidade de fala ${ }^{12}$.
Até o presente momento não havia estudos na área que correlacionassem o Grau de Inteligibilidade de Fala com os déficits em Habilidades Sociais de Comunicação, especificamente, eles apenas expõem que os indivíduos que apresentam diminuição no GIF, também demonstram dificuldades nas interações sociais ${ }^{12}$. No presente estudo pode-se indicar as categorias de HSC que encontram-se deficitárias em crianças com diagnóstico de Desvio Fonológico, favorecendo a elaboração de instrumentos para avaliação e intervenção, permitindo assim, um melhor planejamento para cada caso. 


\section{CONCLUSÃO}

Dentro das limitações desse estudo, pode-se concluir que, o gênero masculino apresentou maior gravidade de Desvio Fonológico, assim como as crianças com maior idade cronológica apresentou menor grau de Desvio Fonológico indo de acordo com grande parte dos estudos na área. E como resultados inovadores na área, pode-se concluir que houve correlação moderada e positiva quanto ao Grau de Inteligibilidade de Fala e dificuldades em Habilidades Sociais de Comunicação e que quanto menor o Grau de Inteligibilidade de Fala, ou seja, maior quantidade de processos fonológicos na fala, maior será o prejuízo nas relações interpessoais da criança.

\begin{abstract}
Purpose: to analyze the relationship between the degree of speech intelligibility and social communication skills in children diagnosed with phonological disorders. Method: the study included 10 children diagnosed with phonological disorders, with an average chronological age of 7.28 years and three females and seven males, performing speech-language therapy twice a week in a nursing school in a medium-sized town in the state of São Paulo. The children were assessed by the phonology of the ABFW instrument, and the severity of phonological disorder was based on the Percentage of Consonants Correct (PCC). And then they were video-typed in interaction structures situations in such a way as to obtain a sample of spontaneous speech, in order to characterize the degree of speech intelligibility (GIF), in addition to observing the presence or absence of the components of social communication skills (HSC). In order to examine the relationship between GIF and HSC we used the Spearman coefficient. Results: it was possible to characterize HSC classes that are deficient in children with phonological diagnostic, and to check a significant and positive relationship between GIF and HSC, showing that the higher GIF is the greater are the difficulties in HSC. Conclusion: this study examined the relationship between GIF and HSC, and identified classes of HSC that are deficient in children diagnosed with phonological disorders, promoting the development of instruments for assessment and intervention, thus allowing better planning for each case.
\end{abstract}

KEYWORDS: Communication; Language Development; Child Language; Interpersonal Relations

\section{REFERÊNCIAS}

1. Borges LC, Salomão NMR. Aquisição da linguagem: Considerações da Perspectiva da Interação Social. Psicologia: Reflexão e Crítica. 2003; 16(2):327-36.

2. Gonçalves GF, Keske-Soares M, Checalin MA. Estudo do papel do contexto linguístico no tratamento do desvio fonológico. Rev Soc Bras Fonoaudiol. 2010; 15(1):96-102.

3. Keske-Soares M, Mota HB, Pagliarin KC, Ceron Ml. Estudos sobre ambientes favoráveis à produção da líquida não-lateral /r/ no tratamento do desvio fonológico. Rev Soc Bras Fonoaudiol. 2007; 12(1):48-54.

4. Papp ACCS, Wertzner HF. O aspecto familial e o transtorno fonológico. Pró-Fono Revista de Atualização Científica, Barueri (SP). 2006; 18(2): 151-60.

5. Telles MS, Macedo CS. Relação entre desenvolvimento motor corporal e aquisição de habilidades orais. Pró-Fono Revista de Atualização Científica. 2008 abr-jun;20(2): 117-22.

6. Casella LA. Relação entre Inteligibilidade de fala e Processos Fonológicos. 2002. Monografia (Graduação em Fonoaudiologia) - Universidade Tuiuti do Paraná - UTP, Curitiba, 2002.

7. Donicht G, Lamprecht RR. Desvio fonológico grave e alterações no sistema vocálico: estudo de um caso. 2009, IV Mostra de Pesquisa da Pós-Graduação - PUCRS.

8. Ghisleni MRL, Keske-Soares M, Mezzomo CL. O uso das estratégias de reparo, considerando a gravidade do desvio fonológico evolutivo. Rev. CEFAC. 2010, Set-Out; 12(5):766-71.

9. Muniz LF, Roazzi A, Schochat E, Teixeira CF, Lucena JA. Avaliação da habilidade de resolução temporal, com uso do tom puro, em crianças com e sem desvio fonológico. Rev CEFAC. 2007; 9(4):550-62.

10. Pagliarin KC, Keske-Soares M, Mota HB. Terapia fonológica em irmãos com diferentes graus 
de gravidade do desvio fonológico. Rev. CEFAC. 2009, Jan-Mar; 11(1): 20-4.

11. Fernandes FDM, Miilher LP. Relações entre a Autistic Behavior Checklist (ABC) e o perfil funcional da comunicação no espectro autístico. Pró-Fono Revista de Atualização Científica. 2008, abr-jun; 20(2):111-6.

12. Yavas M, Hernandorene CM, Lamprech RR. Avaliação fonológica da criança: reeducação e terapia. $1^{\text {a }}$ edição, Porto Alegre: ArtMed, 2001, $148 p$.

13. Payão LMC. Desvios fonológicos em crianças da Educação Infantil: uma análise a partir da hierarquia dos traços distintivos. In: Denilda Moura, organizador. Os desafios da língua: pesquisas em língua falada e escrita. Maceió: Edufal. 2008;1: 341-3.

14. Tavares JG, Payão LMC. Análise da fala de uma criança com desvio fonológico: estudo de caso clínico. J Bras Fonoaudiol. 2006; 6(24): 51-9.

15. Wertzner HF, Pagan LO, Gálea DES, Papp ACCS. Características fonológicas de crianças com transtornos fonológicos com e sem histórico de otite média. Rev Soc Bras Fonoaudiol. 2007; 12(1): 41-7. 16. Athayde ML, Carvalho Q, Mota HB. Vocabulário expressivo de crianças com diferentes níveis de gravidade de desvio fonológico. São Paulo. Rev. CEFAC. 2009;11(supl.2):161-8.

17. Souza PR, Marques JM; Scott LC. Validação de itens para uma escala de avaliação da inteligibilidade de fala. Pró-Fono Revista de Atualização Científica. 2010, jul-set;22(3): 325-32.

18. Shriberg LD, Kwiatkowski J. Phonological disorders I: A diagnostic classification system. Journal of Speech and Hearing Disorders. 1982a;47:226-41.

19. Blanco APF. Caracterização do grau de severidade do desvio fonológico a partir de índices de substituição e omissão. 2002. 70f. Monografia de Especialização - Universidade Federal de Santa Maria, Santa Maria.

20. Lowe RJ. Fonologia - Avaliação e intervenção: Aplicações na Patologia da fala. Porto Alegre: Artes Médicas, 1996, 237p.

http://dx.doi.org/10.1590/S1516-18462011005000106

RECEBIDO EM: 14/03/2011

ACEITO EM: 04/05/2011

Endereço para correspondência:

Fabiana Cristina Carlino

Rua Marcolino Lopes Barreto, 2886

Vila Costa do Sol - São Carlos - SP

CEP: 13566-210

E-mail: fccarlino.usp@gmail.com
21. Barreto SS, Ortiz KZ. Influência da velocidade articulatória e da intensidade na inteligibilidade de fala. Pró-Fono Revista de Atualização Científica. 2008, abr-jun; 20(2):87-92.

22. Donicht G, Pagliarin KC, Mota HB, KeskeSoares M. A inteligibilidade do desvio fonológico julgada por três grupos de julgadores. Pró-Fono Revista de Atualização Científica. 2009, jul-set; 21(3): 213-8.

23. Andrade CRF, Béfi-Lopes DM, Fernandes FDM, Wertzner WH. ABFW: Teste de linguagem infantil nas áreas de Fonologia, Vocabulário, Fluência e Pragmática. Carapicuiba (SP): Pró-Fono, 2004. $90 \mathrm{p}$.

24. Hersen M, Barlow DH. Single case experimental designs: strategies for studying behavior change. $2^{\circ}$ edição, New York: Pergamon Press, 1982.

25. Keske-Soares M, Brancalione AR, Marini C, Pagliarin KC, Ceron MI. Eficácia da terapia para desvios fonológicos com diferentes modelos terapêuticos. Pró-Fono. 2008; 20(3):153-8.

26. Lazzarotto C. Avaliação e planejamento fonoterapêutico para casos de desvio fonológico com base na teoria da otimidade. 2005. 193f. Dissertação (Mestrado em Letras. Área de Concentração Lingüística Aplicada) - Universidade Católica de Pelotas, Pelotas.

27. Carvalho LRL, Mecca FFN, Lightig I. Avaliação das habilidades de metarrepresentação em crianças de sete a oito anos. Pró-Fono Revista de Atualização Científica. 2008, abr-jun;20(2):81-6.

28. César AM, Maksud SS. Caracterização da demanda de fonoaudiologia no serviço público municipal de Ribeirão da Neves - MG. Rev CEFAC. 2007;9(1):133-8.

29. Ceron MI, Keske-Soares M. Terapia fonológica: a generalização a itens não utilizados no tratamento (outras palavras). São Paulo, Revista Cefac. 2007,out-dez;9(4):453-60.

30. Choudhury N, Benasich AA. A family aggregation study: the influence of family history and other risk factors on language development. J Speech Lang Hear Res. 2003; 46(2):261-72.

31. Goulart BNG, Chiari BM. Prevalência de desordens de fala em escolares e fatores associados. Rev. Saúde Pública. 2007;41(5):726-31.

32. Patah LK, Takiuchi N. Prevalência das alterações fonológicas e uso dos process os fonológicos em escolares aos 7 anos. Rev CEFAC. 2008;10(2):158-67.

33. Nunes DA, Payão LMC, Costa RCC. Desvios fonológicos na educação infantil. Rev. CEFAC. 2010, Mar-Abr; 12(2):331-6.

34. Lamprecht RR. Sobre os desvios fonológicos. In: Lamprecht RR, et al. Aquisição fonológica do português: perfil do desenvolvimento e subsídios para terapia. Porto Alegre: Artmed; 2004, p.193-212. 